\title{
The Effects of Variable Viscosity and Thermal Conductivity on MHD Oscillatory Free Convective Flow past a Vertical Plate in Slip Flow Regime with Variable Suction and Periodic Plate Temperature
}

\author{
M. Choudhury ${ }^{1 \dagger}$ and G. C. Hazarika ${ }^{2}$ \\ ${ }^{1}$ Department of Mathematics, N. N. S. College, Assam, 785630, India \\ ${ }^{2}$ Department of Mathematics, Dibrugarh University, Dibrugarh, Assam, 786004, India
}

$\dagger$ Corresponding Author Email: mirabpgc@gmail.com

(Received August 11, 2011; accepted January 27, 2012)

\begin{abstract}
The effects of variable viscosity and thermal conductivity on free convective oscillatory flow of a viscous incompressible and electrically conducting fluid past a vertical plate in slip flow regime with periodic plate temperature when suction velocity oscillates in time about a constant mean is discussed. The fluid viscosity and thermal conductivity are assumed to be inverse linear functions of temperature. The problem is governed by a coupled non-linear system of partial differential equations. Explicit finite difference method is employed to solve the equations. The effects of viscosity variation parameter, thermal conductivity variation parameter, magnetic parameter on the velocity distribution and temperature distribution for $P r_{\infty}=0.7$ fluid and for rarefaction parameter $h=0$ and $h=0.4$ are discussed and shown graphically. Also the effects of these parameters on the skin friction coefficient and on the rate of heat transfer are calculated.
\end{abstract}

Keywords: MHD, natural convection, variable viscosity, variable thermal conductivity.

\section{NOMENCLATURE}

constants

suction parameter

uniform magnetic field strength

constants

local skin-friction coefficient

specific heat at constant pressure

Eckert number

acceleration due to gravity $\left[\mathrm{ms}^{-2}\right]$

Grashof number

rarefaction parameter

thermal conductivity $\left[\mathrm{Wm}^{-1} \mathrm{~K}\right]$

Hartman number

local Nusselt number

fluid pressure $[\mathrm{kPa}]$

Prandtl number

time $[\mathrm{s}]$

temperature $[\mathrm{K}]$

constant

constant

velocity components in the $x$ - and $y$ directions $\left[\mathrm{ms}^{-1}\right]$ $u_{1}, u_{2} \quad$ real and imaginary parts of $u$

$v_{0} \quad$ steady suction velocity

$x, y \quad$ axis in the direction along and normal to the surface respectively

$\alpha \quad$ surface wave amplitude

$\beta \quad$ coefficient of thermal expansion $\left[\mathrm{K}^{-1}\right]$

$\gamma \quad$ constant

$\sigma \quad$ electrical conductivity

$\mu \quad$ coefficient of dynamic viscosity [ $\mathrm{Nsm}^{-2}$ ]

$\xi \quad$ constant

$\rho$ density $\left[\mathrm{kgm}^{-3}\right]$

$\omega$ frequency parameter

$\varepsilon \quad$ amplitude

the kinematic viscosity $\left[\mathrm{m}^{2} \mathrm{~s}^{-1}\right]$

dimensionless temperature

thermal conductivity variation parameter

viscosity variation parameter

ambient free-stream values

wall surface conditions

dimensional quantity 


\section{INTRODUCTION}

Unsteady oscillatory free convective flows play an important role in chemical engineering, turbo machinery and aerospace technology. Such flows arise due to either unsteady motion of a boundary or boundary temperature. The unsteadiness may also be due to oscillatory free stream velocity or temperature. One important class of time dependent viscous flow problems initiated by Lighthill (1954) deals with the effects of unsteady fluctuations of the main stream velocity on the flow of an incompressible fluid past two dimensional bodies.

Many researchers have worked on fluctuating flows of viscous incompressible fluid past an infinite plate. In all these papers the plate was assumed to be maintained at constant temperature. In many practical applications the particle adjacent to a solid surface no longer takes the velocity of the surface. The particle at the surface has a finite temperature of the surrounding stationary fluid. But in many applications quite often the plate temperature starts oscillating about a non-zero mean temperature. The free-convection flow is enhanced by superimposing oscillating temperature on the mean plate temperature. The transient free convection flow past an infinite vertical plate with periodic temperature variation was investigated analytically by Das et al. (1998) Soundalgekar and Wavre (1977) and (1977) studied the unsteady free convection flow past an infinite vertical plate and mass transfer with constant / variable suction.

In many practical applications the particle adjacent to a solid surface no longer takes the velocity of the surface. The particle at the surface has a finite tangential velocity. It slips along the surface. The flow regime is called the slip flow regime and this effect cannot be neglected (Sharma 2003)When the gas is slightly rarefied, results arguing with the observed physical phenomena can be obtained by solving the usual Navier-Stokes equations together with modified boundary conditions allowing for a velocity slip and temperature jump at the surface. This scheme of theoretical investigation of the so called slip-flow regime is particularly suitable for studying the effects of rarefaction on any classical viscous flow problem. Reddy (1964) extended the work of Stuart (1955) for the viscous fluctuating flow over an infinite plate with suction by introducing the slip flow boundary condition in place the of no-slip boundary condition. He observed that the slip boundary condition exhibited a subduing influence on the response of the skin-friction and heat transfer at the wall to the main stream fluctuation. Using this assumption Sharma and Chaudhary (2003) had discussed the free convection flow past a vertical plate in slip-flow regime and also discussed various applications for engineering purposes. Ahmed and Kalita (2008) investigated the effect of the transverse magnetic field and suction parameter on the oscillatory free convective flow past a vertical plate in slip flow regime with variable suction and periodic plate temperature. Very recently Singh and Kumar (2011) investigated the effects of radiation and first order chemical reaction on an unsteady MHD free convective heat and mass transfer flow past a vertical porous flat plate in slip-flow regime when suction velocity oscillates in time about a non-zero constant mean.

Fluid viscosity and thermal conductivity (hence thermal diffusivity) play an important role in the flow characteristic of laminar boundary layer problems. Fluid properties are significantly affected by the variation of temperature. The increase in temperature leads to a local increase in the transport phenomena by reducing the viscosity across the momentum boundary layer and so the heat transfer rate at the wall is affected. In the cooling of electronic equipments, it is relatively frequent to find circumstances in which variable property effects are significant and cannot be neglected. Eswara and Bommaih (2004) established that like other thermophysical properties, temperature dependent viscosity vital role in surface friction and heat transfer rate near the wall. Hazarika and Lahkar (1997) observed that a significant variation takes place in velocity and temperature distribution with the variation of the viscosity and thermal conductivity parameters. Hossain et al (2002) studied natural convection of fluid with variable viscosity from a heated vertical wavy surface.

In all the above works, the Prandtl Number which is a function of viscosity and thermal conductivity is assumed to be constant across the boundary layer. Pantokratoras (2005) and (2007) and Rahman et al. (2009) found that this assumption lead to unrealistic result in modeling the thermal boundary layer flow with a temperature-dependent viscosity. It must be treated as a variable rather than a constant within the boundary layer. The results obtained by Pantokratoras (2005) are different from those existing in the literature, which have been obtained with the assumption of constant Prandtl number.

The objective of this paper is to study the effects of variable viscosity and thermal conductivity on free convective oscillatory flow of a viscous incompressible and electrically conducting fluid past a vertical plate in slip flow regime with periodic plate temperature when suction velocity oscillates in time about a constant mean. The fluid viscosity and thermal conductivity are assumed to be inverse linear functions of temperature and hence considering the variation of Prandtl number across the boundary layer.

\section{Formulation Of The Problem}

An unsteady free-convective flow of a viscous incompressible fluid past an infinite vertical porous plate in slip flow regime with periodic temperature when variable suction velocity distribution fluctuating with time is of the form $v=-v_{0}\left(1+\varepsilon A e^{i \omega t}\right)$ (Ahmed and Kalita 2008), is considered. The $x^{*}$ - axis is taken along the plate with direction opposite to the gravity and $y^{*}$ - axis is taken to be normal to the surface. The velocity components of the fluid are $u^{*}$ and $v^{*}$ in the $x^{*}$ and $y^{*}$ directions respectively, taken parallel and perpendicular to the plate. A magnetic field $B_{0}(x)$ of uniform strength is imposed transversely to the direction of the flow. The magnetic Reynolds Number of the flow is taken to be small enough so that the induced magnetic field can be 
neglected. The fluid property variations with temperature are limited to
i. viscosity,
ii. thermal conductivity and
iii. density.

Following Lai and Kulacki (1990) the fluid viscosity is assumed to be inverse linear function of temperature as

$$
\begin{aligned}
& \frac{1}{\mu}=\frac{1}{\mu_{\infty}}\left[1+\gamma\left(T-T_{\infty}\right)\right]=a\left(T-T_{r}\right) \\
& a=\frac{\gamma}{\mu_{\infty}}, T_{r}=T_{\infty}-\frac{1}{\gamma}
\end{aligned}
$$

$a$ and $T_{r}$ are constants and their values depend on the reference state and thermal property of the fluid. In general $a>0$ for liquids and $a<0$ for gases.

$\gamma$ is a constant based on thermal property of the fluid. For $\gamma \rightarrow 0, \mu=\mu_{\infty}$ (constant).

Also the variation of thermal conductivity is considered as follows (Hazarika and Lahkar 1997)

$$
\begin{aligned}
& \frac{1}{k}=\frac{1}{k_{\infty}}\left[1+\xi\left(T-T_{\infty}\right)=c\left(T-T_{k}\right)\right] \\
& c=\frac{\xi}{k_{\infty}}, T_{k}=T_{\infty}-\frac{1}{\xi}
\end{aligned}
$$

where $c$ and $T_{k}$ are constants and their values depend on the reference state and thermal property of the fluid. $\xi$ is a constant based on thermal property of the fluid. $c>0$ for liquids and $c<0$ for gases.

The influence of variation of density with temperature is restricted to the body force term only, in accordance with the Boussinesq approximation. Since the plate is considered infinite in the $x^{*}$-direction, hence all physical quantities will be independent of $x^{*}$. Under this assumption, the physical variables are functions of $y^{*}$ and $t^{*}$. Thus the equations which govern the freeconvective unsteady hydromagnetic flow are:

$$
\begin{aligned}
& \frac{\partial v^{*}}{\partial y^{*}}=0 \Rightarrow v^{*}=-v_{0}\left(1+\varepsilon A e^{i \omega^{*} t^{*}}\right) \\
& \frac{\partial u^{*}}{\partial t^{*}}-v_{0}\left(1+\varepsilon A e^{i \omega^{*} t^{*}}\right) \frac{\partial u^{*}}{\partial y^{*}} \\
& =\frac{1}{\rho_{\infty}} \frac{\partial}{\partial y^{*}}\left(\boldsymbol{\mu} \frac{\partial u^{*}}{\partial y^{*}}\right)-\frac{\sigma B_{0}^{2} u^{*}}{\rho}+g \boldsymbol{\beta}\left(T-T_{\infty}\right) \\
& c_{p}\left[\frac{\partial T^{*}}{\partial t^{*}}-v_{0}\left(1+\varepsilon A e^{i \omega^{*} t^{*}}\right) \frac{\partial T}{\partial y^{*}}\right] \\
& =\frac{1}{\rho_{\infty}} \frac{\partial}{\partial y^{*}}\left(k \frac{\partial T}{\partial t^{*}}\right)+\frac{\mu}{\rho_{\infty}}\left(\frac{\partial u^{*}}{\partial y^{*}}\right)^{2}
\end{aligned}
$$

The boundary conditions are

$$
\begin{aligned}
& u^{*}=h^{*}\left(\frac{\partial u^{*}}{\partial y^{*}}\right), T=T_{w}+\varepsilon\left(T_{w}-T_{\infty}\right) e^{i \omega^{*} t^{*}} \text { at } y^{*}=0 \\
& u^{*} \rightarrow 0, T \rightarrow T_{\infty} \text { as } y^{*} \rightarrow \infty
\end{aligned}
$$

where $g$ is the acceleration due to gravity, $T$ is the fluid temperature, $\sigma$ is the electrical conductivity, $\rho$ is the fluid density, $c_{p}$ is the specific heat of the fluid at constant pressure, $\mu$ is the fluid viscosity, $k$ is the thermal conductivity of the fluid, $\beta$ is the thermal expansion coefficient, $\varepsilon(<<1)$ is the amplitude, $v_{0}$ is the steady suction velocity (normal) at the plate, $h^{*}$ is the Rarefaction parameter, $A$ is the suction parameter, $\omega^{*}$ is the frequency parameter, the subscripts $\infty$ denotes the free stream condition, and $w$ denotes condition at the wall respectively.

We introduce the following non-dimensional quantities

$$
\begin{aligned}
& y=\frac{y^{*} v_{0}}{\vartheta_{\infty}}, t=\frac{t^{*} v_{0}^{2}}{4 \vartheta_{\infty}}, \omega=\frac{4 \vartheta_{\infty} \omega^{*}}{v_{0}^{2}}, \\
& u=\frac{u^{*}}{v_{0}}, \theta=\frac{T-T_{\infty}}{T_{w}-T_{\infty}}, G_{r}=g \beta \vartheta_{\infty} \frac{T-T_{\infty}}{v_{0}^{3}}, \\
& M=\frac{\sigma B_{0}^{2} \vartheta_{\infty}}{\rho_{\infty} v_{0}^{2}}, h=\frac{v_{0} h^{*}}{\vartheta_{\infty}}, E_{c}=\frac{v_{0}^{2}}{c_{p}\left(T_{w}-T_{\infty}\right)}, \\
& \operatorname{Pr}_{\infty}=\frac{\mu_{\infty} c_{p}}{k_{\infty}}, \vartheta_{\infty}=\frac{\mu_{\infty}}{\rho_{\infty}}
\end{aligned}
$$

Using the transformation Eq. (9), Eq. (6) and Eq. (7) reduces to the following non-dimensional form,

$$
\begin{array}{r}
\frac{1}{4} \frac{\partial u}{\partial t}-\left(1+\varepsilon A e^{i \omega t}\right) \frac{\partial u}{\partial y}=\frac{\theta_{r}}{\left(\theta_{r}-\theta\right)^{2}} \frac{\partial u}{\partial y} \frac{\partial \theta}{\partial y} \\
+G_{r} \theta+\frac{\theta_{r}}{\theta_{r}-\theta} \frac{\partial^{2} u}{\partial y^{2}}-M u \\
\frac{1}{4} \frac{\partial \theta}{\partial t}-\left(1+\varepsilon A e^{i \omega t}\right) \frac{\partial \theta}{\partial y}=\frac{1}{P_{r}} \frac{\theta_{k}}{\left(\theta_{k}-\theta\right)^{2}}\left(\frac{\partial \theta}{\partial y}\right)^{2} \\
+\frac{1}{\operatorname{Pr}_{\infty}} \frac{\theta_{k}}{\theta_{k}-\theta} \frac{\partial^{2} \theta}{\partial y^{2}}+E_{c} \frac{\theta_{r}}{\theta_{r}-\theta}\left(\frac{\partial u}{\partial y}\right)^{2}
\end{array}
$$

where $\vartheta_{\infty}$ is the kinematic viscosity, $G_{r}$ is the Grashof Number, $M$ is the Hartmann Number, $h$ is the dimensionless rarefaction parameter, $E_{c}$ is the Eckert number, $P r_{\infty}$ is the ambient Prandtl number,

$\theta_{r}=\frac{T_{r}-T_{\infty}}{T_{w}-T_{\infty}}$ is the viscosity variation parameter and $\theta_{k}=\frac{T_{k}-T_{\infty}}{T_{w}-T_{\infty}} \quad$ is the thermal conductivity variation parameter.

The Prandtl number $\operatorname{Pr}=\frac{\mu c_{p}}{k}$ is a function of viscosity as well as thermal conductivity and as both the viscosity and thermal conductivity varies across the boundary layer, the Prandtl number also varies. The assumption of constant Prandtl number may produce unrealistic results (Rahman et al. 2009) Therefore, the variable Prandtl number related to the variable viscosity and variable thermal conductivity is defined as

$$
\operatorname{Pr}=\frac{\mu c_{p}}{k}=\frac{\theta_{r}}{\theta_{r}-\theta} \frac{\theta_{k}-\theta}{\theta_{k}} \operatorname{Pr}_{\infty}
$$

Using Eq. (12), the non-dimensional temperature Eq. (11) can be written as 


$$
\begin{aligned}
& \frac{1}{4} \frac{\partial \theta}{\partial t}-\left(1+\varepsilon A e^{i \omega t}\right) \frac{\partial \theta}{\partial y} \\
& =\frac{1}{\operatorname{Pr}} \frac{\theta r}{\left(\theta_{r}-\theta\right)\left(\theta_{k}-\theta\right)}\left(\frac{\partial \theta}{\partial y}\right)^{2} \\
& +\frac{1}{\operatorname{Pr}} \frac{\theta r}{\theta_{r}-\theta} \frac{\partial^{2} \theta}{\partial y^{2}}+E_{c} \frac{\theta_{r}}{\theta_{r}-\theta}\left(\frac{\partial u}{\partial y}\right)^{2}
\end{aligned}
$$

which is the corrected non-dimensional form of the energy equation for modeling thermal boundary layer flows with temperature dependent viscosity and thermal conductivity.

The corresponding boundary conditions in nondimensional form

$$
\begin{aligned}
& y=0 ; \quad u=h \frac{\partial u}{\partial y}, \theta=1+\varepsilon \mathrm{e}^{\mathrm{i} \omega t} \\
& y \rightarrow \infty ; \quad u=0, \theta=0
\end{aligned}
$$

\section{Solution Of The Problem}

In order to solve the differential Eq. (10) and Eq. (13) we assume that:

$$
\begin{aligned}
& u=u_{1}+i u_{2} \\
& \theta=\theta_{1}+i \theta_{2}
\end{aligned}
$$

By substituting Eq. (15) and Eq. (16) into Eq. (10) and Eq. (13) and equating real and imaginary parts we get the following system of differential equation:

$$
\begin{aligned}
& \frac{1}{4} \frac{\partial u_{1}}{\partial t}-(1+\varepsilon A \cos \omega t) \frac{\partial u_{1}}{\partial y}+\varepsilon \operatorname{Asin} \omega t \frac{\partial u_{2}}{\partial y} \\
& =G_{r} \theta_{1}-M u_{1} \\
& +\frac{\theta_{r}}{\left(\theta_{r}-\theta_{1}\right)^{2}+\theta_{2}^{2}}\left(\left(\theta_{r}-\theta_{1}\right) \frac{\partial^{2} u_{1}}{\partial y^{2}}-\theta_{2} \frac{\partial^{2} u_{2}}{\partial y^{2}}\right) \\
& +\frac{\theta_{r}}{\left(\left(\theta_{r}-\theta_{1}\right)^{2}+\theta_{2}^{2}\right)^{2}} \\
& \left\{\begin{array}{c}
\left(\left(\theta_{r}-\theta_{1}\right)^{2}-\theta_{2}^{2}\right)\left(\frac{\partial u_{1}}{\partial y} \frac{\partial \theta_{1}}{\partial y}-\frac{\partial u_{2}}{\partial y} \frac{\partial \theta_{2}}{\partial y}\right) \\
-2 \theta_{2}\left(\theta_{r}-\theta_{1}\right)\left(\frac{\partial u_{2}}{\partial y} \frac{\partial \theta_{1}}{\partial y}+\frac{\partial u_{1}}{\partial y} \frac{\partial \theta_{2}}{\partial y}\right)
\end{array}\right\}
\end{aligned}
$$

$$
\begin{aligned}
& \frac{1}{4} \frac{\partial u_{2}}{\partial t}-(1+\varepsilon A \cos \omega t) \frac{\partial u_{2}}{\partial y}-\varepsilon A \sin \omega t \frac{\partial u_{1}}{\partial y}=G_{r} \theta_{2}+ \\
& \frac{\theta_{r}}{\left(\theta_{r}-\theta_{1}\right)^{2}+\theta_{2}^{2}}\left(\left(\theta_{r}-\theta_{1}\right) \frac{\partial^{2} u_{2}}{\partial y^{2}}+\theta_{2} \frac{\partial^{2} u_{1}}{\partial y^{2}}\right)-M u_{2}+ \\
& \frac{\theta_{r}}{\left(\left(\theta_{r}-\theta_{1}\right)^{2}+\theta_{2}^{2}\right)^{2}} \\
& \left\{\begin{array}{c}
\left(\left(\theta_{r}-\theta_{1}\right)^{2}-\theta_{2}^{2}\right)\left(\frac{\partial u_{2}}{\partial y} \frac{\partial \theta_{1}}{\partial y}+\frac{\partial u_{1}}{\partial y} \frac{\partial \theta_{2}}{\partial y}\right) \\
+2 \theta_{2}\left(\theta_{r}-\theta_{1}\right)\left(\frac{\partial u_{1}}{\partial y} \frac{\partial \theta_{1}}{\partial y}-\frac{\partial u_{2}}{\partial y} \frac{\partial \theta_{2}}{\partial y}\right)
\end{array}\right\}
\end{aligned}
$$

$$
\begin{aligned}
& \frac{1}{4} \frac{\partial \theta_{1}}{\partial t}-(1+\varepsilon A \cos \omega t) \frac{\partial \theta_{1}}{\partial y}+\varepsilon A \sin \omega t \frac{\partial \theta_{2}}{\partial y} \\
& =\frac{1}{\operatorname{Pr}} \frac{\theta r}{\left(\theta_{r}-\theta_{1}\right)^{2}+\theta_{2}^{2}}\left(\left(\theta_{r}-\theta_{1}\right) \frac{\partial^{2} \theta_{1}}{\partial y^{2}}-\theta_{2} \frac{\partial^{2} \theta_{2}}{\partial y^{2}}\right)+ \\
& \frac{1}{\operatorname{Pr}} \overline{\left(\left(\theta_{r}-\theta_{1}\right)^{2}+\theta_{2}^{2}\right)\left(\left(\theta_{k}-\theta_{1}\right)^{2}+\theta_{2}^{2}\right)} \\
& \left.\left\{\begin{array}{l}
\left(\left(\theta_{r}-\theta_{1}\right)\left(\theta_{k}-\theta_{1}\right)-\theta_{2}^{2}\right) \\
\left(\left(\frac{\partial \theta_{1}}{\partial y}\right)^{2}-\left(\frac{\partial \theta_{2}}{\partial y}\right)^{2}\right) \\
-2 \theta_{2}\left(\theta_{r}+\theta_{k}-2 \theta_{1}\right) \\
\frac{\partial \theta_{1}}{\partial y} \frac{\partial \theta_{2}}{\partial y}
\end{array}\right\} \begin{array}{l}
\left(\theta_{r}-\theta_{1}\right)\left(\left(\frac{\partial u_{1}}{\partial y}\right)^{2}-\left(\frac{\partial u_{2}}{\partial y}\right)^{2}\right) \\
-2 \theta_{2} \frac{\partial u_{1}}{\partial y} \frac{\partial u_{2}}{\partial y}
\end{array}\right\} \\
& +E_{c} \frac{\theta_{r}}{\left(\theta_{r}-\theta_{1}\right)^{2}+\theta_{2}^{2}}\{
\end{aligned}
$$

$$
\frac{1}{4} \frac{\partial \theta_{2}}{\partial t}-(1+\varepsilon A \cos \omega t) \frac{\partial \theta_{2}}{\partial y}-\varepsilon A \sin \omega t \frac{\partial \theta_{1}}{\partial y}
$$$$
=\frac{1}{\operatorname{Pr}} \frac{\theta_{r}}{\left(\left(\theta_{r}-\theta_{1}\right)^{2}+\theta_{2}^{2}\right)\left(\left(\theta_{k}-\theta_{1}\right)^{2}+\theta_{2}^{2}\right)}
$$$$
\left\{\begin{array}{c}
\theta_{2}\left(\theta_{r}+\theta_{k}-2 \theta_{1}\right)\left(\left(\frac{\partial \theta_{1}}{\partial y}\right)^{2}-\left(\frac{\partial \theta_{2}}{\partial y}\right)^{2}\right) \\
+2\left(\left(\theta_{r}-\theta_{1}\right)\left(\theta_{k}-\theta_{1}\right)-\theta_{2}^{2}\right) \frac{\partial \theta_{1}}{\partial y} \frac{\partial \theta_{2}}{\partial y}
\end{array}\right\}
$$

$+\frac{1}{\operatorname{Pr}} \frac{\theta_{r}}{\left(\theta_{r}-\theta_{1}\right)^{2}+\theta_{2}^{2}}\left(\left(\theta_{r}-\theta_{1}\right) \frac{\partial^{2} \theta_{2}}{\partial y^{2}}+\theta_{2} \frac{\partial^{2} \theta_{1}}{\partial y^{2}}\right)$

$$
+E_{c} \frac{\theta_{r}}{\left(\theta_{r}-\theta_{1}\right)^{2}+\theta_{2}^{2}}\left\{\begin{array}{l}
2\left(\theta_{r}-\theta_{1}\right) \frac{\partial u_{1}}{\partial y} \frac{\partial u_{2}}{\partial y} \\
+\theta_{2}\left(\left(\frac{\partial u_{1}}{\partial y}\right)^{2}-\left(\frac{\partial u_{2}}{\partial y}\right)^{2}\right)
\end{array}\right\}
$$

The boundary condition Eq. (14) reduces to

$$
\begin{aligned}
& y=0 ; \quad u_{1}=h \frac{\partial u_{1}}{\partial y}, u_{2}=h \frac{\partial u_{2}}{\partial y}, \\
& \theta_{1}=1+\varepsilon \cos \omega t, \theta_{2}=\varepsilon \sin \omega t \\
& y \rightarrow \infty ; u_{1}=0, u_{2}=0, \theta_{1}=0, \theta_{2}=0
\end{aligned}
$$

Equations (17) to (20) are coupled non-linear partial differential equations which are to be solved by using the boundary conditions (Eq. (21)). However, exact or approximate solutions are not possible for this set of equations and hence we solve these equations by finite difference methods. The central difference is used for the diffusion terms and the forward difference scheme is used for the convection terms. After experimenting with a few set of mesh sizes, the mesh sizes were fixed at $\Delta y=0.1$ and $\Delta t=0.01$. The procedure is repeated until $t=1$. This gives sufficient accuracy as the boundary conditions are satisfied. 
The important characteristics of the problem are the skin-friction co-efficient and heat transfer rate at the plate.

The coefficient of skin-friction at the surface is given by

$$
\begin{aligned}
& C_{f}=\frac{\tau}{\rho_{\infty} v_{0}^{2}} \\
& =\text { real part }\left.\frac{\theta_{r}}{\theta_{r}-\theta} \frac{\partial \imath}{\partial y}\right|_{y=0} \\
& \text { where } \boldsymbol{\tau}=\left.\boldsymbol{\mu} \frac{\partial u^{*}}{\partial y^{*}}\right|_{y^{*}=0} .
\end{aligned}
$$

The rate of heat transfer in terms of Nusselt Number at the surface is

$$
\begin{aligned}
& N u=\frac{q_{w} \vartheta}{k_{\infty} v_{0}\left(T_{w}-T_{\infty}\right)} \\
& =- \text { real part }\left.\frac{\theta_{k}}{\theta_{k}-\theta} \frac{\partial T}{\partial y}\right|_{y=0} \\
& \text { where } \quad q_{w}=-\left.k \frac{\partial T}{\partial y^{*}}\right|_{y^{*}=0} .
\end{aligned}
$$

\section{Results AND Discussion}

As a result of the numerical calculations the velocity and temperature distribution for the flow are obtained from Eq. (17) to Eq. (20) which are displayed in figures for different values of $\theta_{r}$ (Viscosity variation parameter), $\theta_{k}$ (Thermal conductivity variation parameter), $M$ (Hartmann number) which are chosen arbitrarily for fluid with Prandtl number $P_{r}=0.71$ (Pantokratoras 2005) and Eckert number $E_{c}=0.01$. In the present analysis the values of Grashof number $G_{r}$ are chosen arbitrarily, we take $G_{r}=5>0$ correspond to the cooling of the plate. Throughout our investigation we take $h$ (rarefaction parameter) $=0$ and 0.4 (Sharma 2003), suction parameter $A=0.2$ (Ahmed and Kalita 2008), frequency parameter (dimensionless) $\omega=1$ (Ahmed and Kalita 2008), and amplitude oscillation $\varepsilon=0.1$ (Ahmed and Kalita 2008). Also the skin-friction coefficient $C_{f}$ and the Nusselt number $\mathrm{Nu}$ are calculated from Eq. (22) and Eq. (23) and are tabulated in Table 1 to Table 3 for same set of parameters.

From Table 1 it is observed that an increasing value of the viscosity parameter $\left(\theta_{r}=3,5 \ldots 15\right)$ leads to decrease in the values of skin friction coefficient $C_{f}$ for rarefaction parameter $h=0.4$ and $\mathrm{h}=0.0$.

Table 2 depicts that the Nusselt number decreases due to increase in the thermal conductivity parameter $\left(\theta_{k}=3\right.$, $5 \ldots 15)$ for $h=0.4$. The results are qualitatively similar to the related study by Rahman et al. (2008) and show that increasing the thermal conductivity of the fluid leads to a decrease in the Nusselt number. This may partly be explained by the fact that that increasing thermal conductivity has the effect of accelerating and increasing the temperature of the fluid.
Table 3 depicts that skin friction coefficient $C_{f}$ the Hartman number $(M=1,2,3,4,5)$ for $h(=0,0.4)$. It is observed that $C_{f}$ increases (Muthucumaraswamy and Vadivel 2004) as $M$ increases.

From Table 1 and Table 3 , it is observed that $C_{f}$ increases as $h(=0,0.4)$ decreases which agrees with Sharma (2003).

Table 1 Effects of viscosity variation parameter $\theta_{r}$ on Skin-friction coefficient $C_{f}$ for $P_{r}=0.71, G_{r}=5, M=0.5$,

\begin{tabular}{|c|c|c|}
\multicolumn{3}{|c|}{$E_{c}=0.01, \theta_{k}=15$} \\
\hline$\theta r$ & $h=0.4$ & $h=0.0$ \\
\hline 3 & 1.578875 & 7.894374 \\
\hline 5 & 1.282022 & 6.410112 \\
\hline 7 & 1.186423 & 5.932116 \\
\hline 9 & 1.139228 & 5.69614 \\
\hline 11 & 1.111101 & 5.555506 \\
\hline 13 & 1.092429 & 5.462145 \\
\hline 15 & 1.07913 & 5.395649 \\
\hline
\end{tabular}

Table 2 Effects of thermal conductivity variation parameter $\theta_{k}$ Nusselt number $N u$ for $P_{r}=0.71, G_{r}=5$,

\begin{tabular}{|c|c|}
\hline$=0.5, E_{c}=0.01, \theta_{r}=15$ \\
\hline$\theta_{k}$ & $h=0.4$ \\
\hline 3 & 1.580396 \\
\hline 5 & 1.282624 \\
\hline 7 & 1.18679 \\
\hline 9 & 1.139491 \\
\hline 11 & 1.111306 \\
\hline 13 & 1.092596 \\
\hline 5 & 1.079271 \\
\hline
\end{tabular}

Table 3 Effects of Hartman Number $M$ on Skin-friction coefficient $C_{f}$ for $P_{r}=0.71, G_{r}=5, \theta_{k}=15, E_{c}=0.01$,

$$
\theta_{r}=15 \text {. }
$$

\begin{tabular}{|c|c|c|}
\hline$M$ & $h=0.4$ & $h=0.0$ \\
\hline 1 & -2.43321 & 3.524447 \\
\hline 2 & -2.25446 & 3.932257 \\
\hline 3 & -2.0673 & 4.351734 \\
\hline 4 & -1.8716 & 4.783027 \\
\hline 5 & -1.66722 & 5.226292 \\
\hline
\end{tabular}

There is no significant variation in Nusselt number $\mathrm{Nu}$ due to variation of viscosity variation parameter $\theta_{r}$ and Hartman number $M$.

The variation of velocity profiles are shown in Fig. 1 for $M=0.5, G_{r}=5$ where $\theta_{r}=5,10,15$ and $h=0.4$. Fig. 2 depicts the velocity profiles for same set of parameters and rarefaction parameter $h=0$. 
Figure 3 and Fig. 4 represent temperature profiles for thermal conductivity variation parameter $\theta_{k}$ for $h=0.4$ and $h=0$ respectively. In both cases, temperature field increases with increasing $\theta_{k}$. The temperature profiles are wider for rarefaction parameter $h=0.4$.

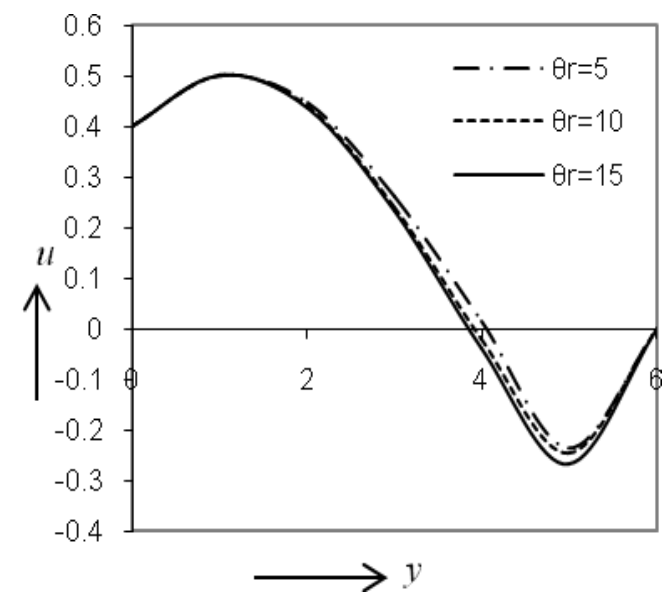

Fig. 1. Velocity Profiles for $M=0.5, G_{r}=5, \theta_{k}=15$, $h=0.4$

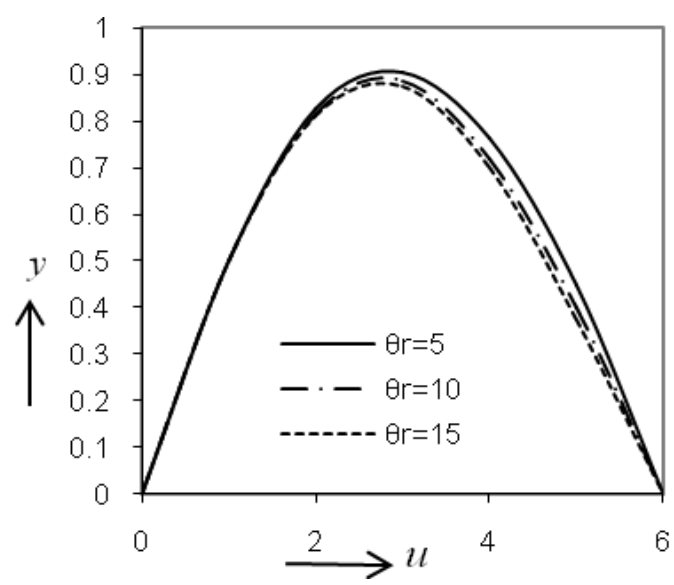

Fig.2. Velocity Profiles for $M=0.5, G_{r}=5, \theta_{k}=15, h=0$.

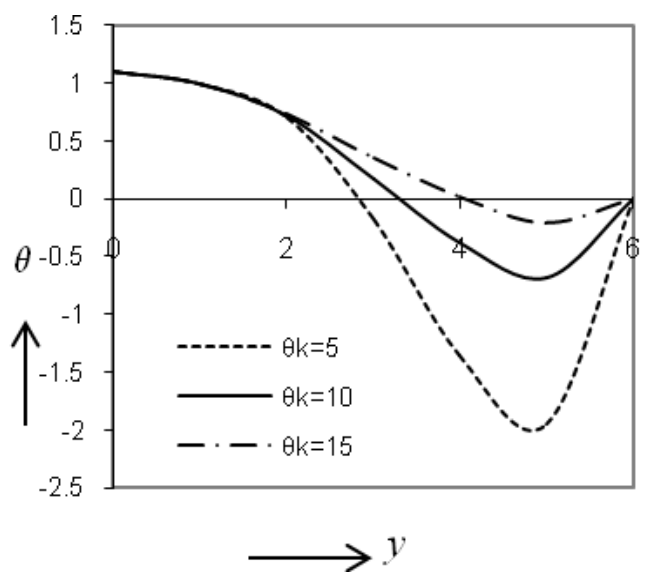

Fig.3. Temperature Profiles for $\theta_{r}=15, G_{r}=5, M=0.5$, $\mathrm{h}=0.4$

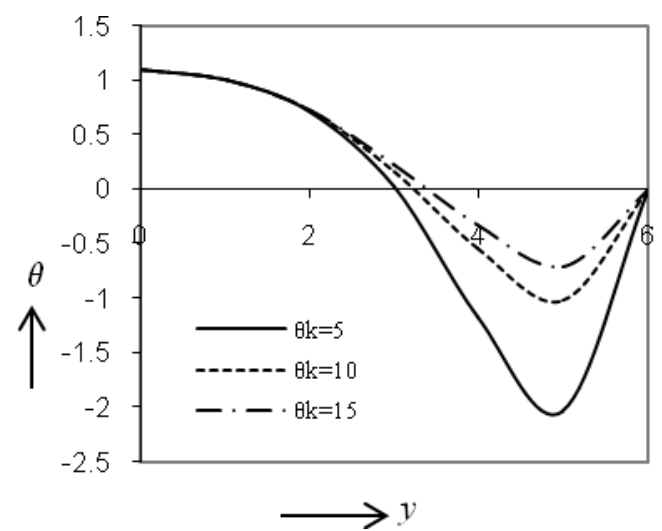

Fig.4. Temperature Profiles for $\theta_{r}=15, G_{r}=5, M=0.5$, $\mathrm{h}=0$

Figure 5 and Fig. 6 represent the variation in velocity distribution with the variation of Hartman number $M$ for rarefaction parameter $h=0.4$ and $h=0$ respectively. These curves show that velocity decreases in the presence of magnetic field which agrees with the expectations, since the magnetic field exerts a retarding force on the free convective flow (Muthucumaraswamy and Vadivel 2004).

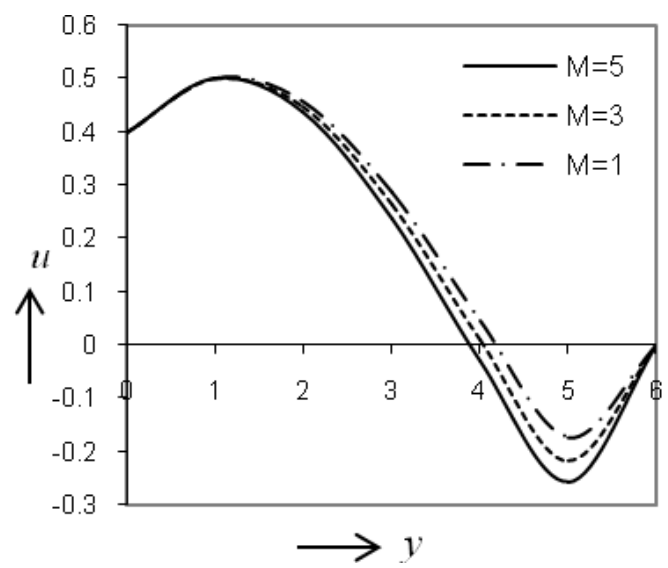

Fig. 5. Velocity Profiles for $\theta_{r}=15, G_{r}=5, \theta_{k}=15$, $h=0.4$.

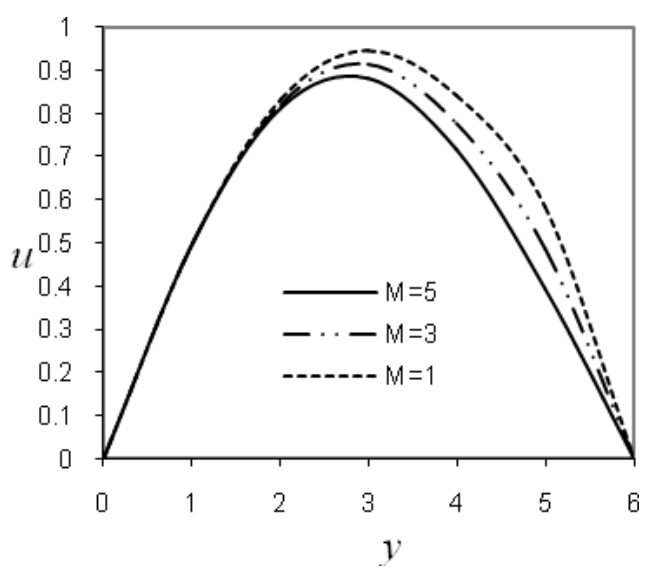

Fig. 6. Velocity Profiles for $\theta_{r}=15, G_{r}=5, \theta_{k}=15$, $h=0.0$ 


\section{CONCLUSION}

The theoretical solution of free convective oscillatory MHD past a vertical plate in slip flow regime with variable suction and periodic plate temperature is considered. Fluid viscosity and thermal conductivity are considered as inverse linear functions of temperature. The presented analysis has shown that for modeling thermal boundary layers with temperature dependent viscosity and thermal conductivity, the Prandtl number must be treated as variable inside the boundary layer. The flow field is appreciably influenced by the viscosity-temperature and thermal conductivitytemperature variation and also by the magnetic parameter.

\section{ACKNOWLEDGEMENTS}

The authors sincerely thank the referee for very useful comments and suggestions in improving the paper

\section{REFERENCES}

Ahmed, N. and D. Kalita (2008). MHD oscillatory freeconvective flow past a vertical plate in slip-flow regime with variable suction and periodic plate temperature. Heat and Technology 26 (2), 85-92.

Das, U. N., R. K. Deka and V. M. Soundalgekar (1998). Transient free convection flow past an infinite vertical plate with periodic temperature variation. Journal of Heat Transfer (ASME) 121, 1091-1094.

Eswara, A.T. and B.T. Bommaih (2004). The effect of variable viscosity on laminar flow due to a point sink. Indian Journal of Pure and Applied Mathematics. 35 (6), 811-815.

Hazarika, G.C. and J. Lahkar (1997). Effects of variable viscosity and thermal conductivity on free convective mass transfer flow past a vertical isothermal cone surface in presence of magnetic field. Mathematical Forum xi, 1-15.

Hossain, M. A., S. Kabir and D.A.S. Rees (2002). Natural convection of fluid with variable viscosity from a heated vertical wavy surface. Zeitschrift für angewandte Mathematik und Physik ZAMP 53(1), 48-57.

Lai, F. C. and F. A. Kulacki (1990). The effect of variable viscosity on convective heat transfer along a vertical surface in a saturated porous medium. International Journal of Heat and Mass Transfer 3(5), $1028-1031$.

Lighthill, M. J. (1954). The response of laminar skin friction and heat transfer to fluctuations in the stream velocity. Proceedings the Royal of Society A 224(1156), 1-23

Muthucumaraswamy, R. and V. U. S. Vadivel (2004). Heat transfer effect on moving vertical surface in the presence of magnetic field. Mathematics Education XXXVIII (1), 20- 29.
Pantokratoras, A. (2007). Non- Darcian forced convection heat transfer over a flat plate in a porous medium with variable viscosity and variable Prandtl number. Journal of Porous Media 10(22), 201-208.

Pantokratoras, A. (2005). Forced and mixed convection boundary layer flow along a flat plate with variable viscosity and variable Prandtl number, new results. Heat and Mass Transfer 41(12), 1085-1094.

Rahman, M. M., M. A. Rahman, M. A. Samad and M. S. Alam (2009). Heat Transfer in a Micropolar Fluid along a Non-linear Stretching Sheet with a Temperature-Dependent Viscosity and Variable Surface Temperature. International Journal of Thermophysics 30(5), 1649-1670.

Rahman, M. M., A. A. Mamun, M. A. Azim and M. A. Alim (2008). Effects of temperature dependent thermal conductivity on MHD free convection flow along a vertical flat plate with heat conduction. Nonlinear Analysis: Modeling and Control 13 (4), $513-524$.

Reddy, K. C. (1964). Fluctuating flow past a porous infinite plate in slip flow regime. Quarterly Journal of Mechanics and Applied Mathematics. 17(4), 381395.

Sharma, P. K. (2005). Influence of periodic temperature and concentration on unsteady free convective viscous incompressible flow and heat transfer past a vertical plate in slip-flow regime. Mathematicas: Ensenanza Universitaria XIII (1), 51-62.

Sharma, P. K. and R. C. Chaudhary (2003). Effect of variable suction on transient free convective viscous incompressible flow past a vertical plate with periodic plate temperature variable in sleep flow regime. EJER 8 (2), 3-38.

Singh, K.D. and R. Kumar (2011). Fluctuating Heat and Mass Transfer on Unsteady MHD Free Convection Flow of Radiating and Reacting Fluid past a Vertical Porous Plate in Slip- Flow Regime. Journal of Applied Fluid Mechanics 4 (4), 101-106.

Soundalgekar, V. M. and P. D. Wavre (1977). Unsteady free convection flow past an infinite vertical plate with constant suction and mass transfer. International Journal of Heat and Mass Transfer 20(12), 1363-1373.

Soundalgekar, V. M. and P. D. Wavre (1977). Unsteady free convection flow past an infinite vertical plate with variable suction and mass transfer. International Journal of Heat and Mass Transfer 20(12), 1375-1380.

Stuart, J. T. (1955). A solution of the Navier-Stokes and energy equations illustrating the response of skin friction and temperature of an infinite plate thermometer to fluctuation in the stream velocity. Proceedings the Royal of Society A 231(1184), 116-130. 